\title{
T-REx: Transcriptome analysis webserver for RNA-seq Expression data
}

\author{
Anne de Jong ${ }^{1,2,3^{*}}$, Sjoerd van der Meulen ${ }^{1}$, Oscar P. Kuipers ${ }^{1,2}$ and Jan Kok ${ }^{1,2}$
}

\begin{abstract}
Background: Transcriptomics analyses of bacteria (and other organisms) provide global as well as detailed information on gene expression levels and, consequently, on other processes in the cell. RNA sequencing (RNA-seq) has over the past few years become the most accurate method for global transcriptome measurements and for the identification of novel RNAs. This development has been accompanied by advances in the bioinformatics methods, tools and software packages that deal with the analysis of the large data sets resulting from RNA-seq efforts.

Results: Based on years of experience in analyzing transcriptome data, we developed a user-friendly webserver that performs the statistical analysis on the gene expression values generated by RNA-seq. It also provides the user with a whole range of data plots. We benchmarked our RNA-seq pipeline, T-REX, using a case study of CodY mutants of Bacillus subtilis and show that it could easily and automatically reproduce the statistical analysis of the cognate publication. Furthermore, by mining the correlation matrices, k-means clusters and heatmaps generated by T-REx we observed interesting gene-behavior and identified sub-groups in the CodY regulon.

Conclusion: T-REx is a parameter-free statistical analysis pipeline for RNA-seq gene expression data that is dedicated for use by biologists and bioinformaticians alike. The tables and figures produced by T-REX are in most cases sufficient to accurately mine the statistical results. In addition to the stand-alone version, we offer a user-friendly webserver that only needs basic input (http://genome2d.molgenrug.nl).
\end{abstract}

\section{Background}

Measuring mRNA levels in cells or tissues is being performed ever since the introduction of Northern blot hybridization. Implementation of DNA-microarray technology has allowed to measure gene expression at a genome-wide scale. Although DNA-microarrays are still being used, the technique is now almost fully replaced by next-generation (RNA) sequencing (RNA-seq). This relatively new method can be used to determine absolute gene expression levels and is far more accurate than DNA-microarraying, which commonly generates ratiobased data. Analysis of RNA-seq data is in principle divided into two stages. The first step involves the quality control and mapping of the sequence reads to an annotated reference genome. Command line tools such as SAMtools [1] and BEDtools [2] are commonly used

\footnotetext{
* Correspondence: anne.de.jong@rug.nl

'Molecular Genetics, University of Groningen, Nijenborgh 7, 9747AG

Groningen, The Netherlands

${ }^{2}$ Top Institute Food and Nutrition (TIFN), Nieuwe Kanaal 9A, 6709PA,

Wageningen, The Netherlands

Full list of author information is available at the end of the article
}

but user friendly software packages such as RockHopper [3] and NGS-Trex [4] have also been developed. This generates gene (RNA) expression values such as Reads Per Kilobase per Million reads (RPKM), Fragments Per Kilobase per Million (FPKMs), Counts Per Million (CPM) or other gene expression units. The second step entails statistical and biological analyses of the transcriptome data using tools such as EdgeR [5], DEseq [6] and others [7]. These investigations could involve the analysis of differential gene expression between two samples, but they can also be more complex such as in the analysis of data obtained from times series experiments or of multiple experiments from multiple time points. To blend the various approaches into one common analysis method, factorial design is the most favorable procedure used for the analysis of DNAmicroarray data (LimmeR, [8]) as well as for RNA-seq data analysis (EdgeR and DEseq). Factorial design offers flexibility in controlling how to perform the statistical analyses. Once the factorial design has been made, six analysis steps are generally executed; i) normalization 
and scaling of the gene expression values, ii) global analysis of the experiments using e.g., Principal Component Analysis (PCA), iii) differential expression of genes between experiments, iv) clustering of genes expression levels and/or ratios between experiments, v) studying the behavior of groups of genes of interest (classes), vi) functional analysis or gene-set enrichment. A variety of software packages can be used to perform the steps mentioned above but, due to issues regarding userfriendliness, these are usually practical mainly for bioinformaticians. The main topics in examining the huge amount of transcriptomics data obtained by RNAseq are the choice of proper data analysis methods, the setting of suitable parameters and the conversion and combining of data generated in the different stages of analysis. The development of the RNA-seq analysis pipeline T-Rex and the choices we made with respect to the methods and parameters employed were based on an iterative process between bioinformaticians and biologists. In this article we introduce and describe this pipeline, T-REx, a user-friendly webserver to analyse RNA-seq-derived gene expression data that has been optimized for prokaryotes. In addition we offer the Rscript, which gives the user full control over the parameters used in the statistical analyses.

\section{Implementation}

The first steps in the statistical analysis of gene expression data are data normalization and determination of the genes that are differentially expressed between samples. To do this, the factorial design statistical method of the RNA-seq analysis R-package EdgeR [5] was chosen. Routines for clustering and plotting of graphics were derived from the open source software repository Bioconductor [9].

The pipeline (Additional file 1 and 2) requires raw RNA expression level data as an input for RNA-seq data analysis. RPKM, FPKM, TPM [10] or any other count values can be combined in one table and used as an input for T-REx. Also, DNA-microarray data containing gene (RNA) expression levels can be used. For the calculation of the $p$-values for differential expression the dispersion model of EdgeR is employed, which is optimized for the use of CPM values. Comparison of the differentially expressed genes using either CPM or RPKM values showed only differences in the TopHits genes close to cutoff values ( $p$-value 0.05 and fold-change of 2 ). The second input file defines the factors that are used to describe the experiments and the replicates (Table 1A). A third file is used to define which comparisons (contrasts) should be made between the various experimental conditions (Table 1B). The researcher is offered an easy and flexible way to produce the results by simply adjusting the contrast file. Although these three files are enough to perform a complete statistical analysis of the dataset under
Table 1 Input files for the RNA-seq analysis pipeline

\begin{tabular}{lllllll}
\hline A) Factors & & & B) Contrasts & C) Classes & \\
\hline Experiment & Strain & Time & A_F71Y-WT & BSU00490 & green & CodY \\
WT1 & WT & T1 & B_R61K-WT & BSU01650 & green & CodY \\
WT2 & WT & T1 & C_R61H-WT & BSU01660 & green & CodY \\
F71Y1 & A_F71Y & T1 & Null-WT & BSU01670 & green & CodY \\
F71Y2 & A_F71Y & T1 & & BSU01680 & green & CodY \\
R61K1 & B_R61K & T2 & & etC... & $\ldots$ & ... \\
R61K2 & B_R61K & T2 & & BSU03981 & red & CcpA \\
R61H1 & C_R61K & T2 & & BSU03982 & red & CcpA \\
R61H2 & C_R61K & T2 & & BSU03990 & red & CcpA \\
null1 & Null & T2 & & BSU04160 & red & CcpA \\
null2 & Null & T2 & & BSU04470 & red & CcpA \\
& & & & etc... & ... & ... \\
\hline
\end{tabular}

A) File describing the experiments and containing information of experiment replicates, B) File with the comparisons (contrasts) to be made, C) File with groups of genes/RNAs of interest

study, the added value of T-REx is a fourth file, which allows focusing the analysis on one group or multiple groups of genes or RNAs (e.g., the regulons of CodY and CcpA in the example given below). To do this, the researcher predefines a Class file of groups of genes of interest (Table 1C. More details, examples and tutorials for creating the 4 files can be obtained from the T-REx webserver.

Once T-REx is fed with the four input files, normalization and global analysis of the data will be performed and visualized in several graphs. These graphs include library sizes, box plots of normalized signals, a correlation matrix of experiments and a two-dimensional Multidimensional Scaling (MDS) plot of the samples to be studied. Although various normalization methods have been developed, we found the trimmed-median mean method (TMM) of EdgeR to be the most accurate for RNA-seq data derived from prokaryotes. Subsequently, statistical analysis of differential expression of genes of all contrasts (derived from Table 1B) is performed and the outcome is visualized in MA and volcano plots. In the MA plots traditionally used in DNA-microarray analysis gene expression is plotted against ratio values while volcano plots compute gene expression ratios against their $p$-values. In both of these dot-plots (in which dots represent genes) each dot gets a color as defined by the user in the Class file (Table 1C) while non-defined dots are colored black, allowing to easily identify the behavior of Class-defined genes. Genes can have an expression value of zero, e.g., when comparing two bacterial species in which one of the genes is absent. To prevent errors as a consequence of having to divide by zero, zero values are scaled to noise level. Thus, these genes will be flagged by a cross-sign in the MA and volcano plots.

K-means clustering $[2,11]$ is considered to be one of the most powerful methods to analyze behavior of gene 
expressions between dependent experiments, such as samples taken over a course of time from the same culture. However, defining the number of clusters in which the data can be divided is arbitrary. To tackle this issue, the analysis pipeline will estimate the number of clusters for a certain data set automatically. The number of genes is furthermore limited to include only those that have a fold change $\geq 2$ and a $p$-value $\leq 0.05$ (TopHits) in at least one of the contrasts. Subsequently, two additional groups of genes are defined, one group containing genes that show highly differential expression in at least one of the contrasts (HighFold: fold change $\geq 5$ and $p$-value $\leq 0.01$ ) and a group from which non changed genes are removed (no_background: fold change $\leq 1.4$ and $p$-value $\geq 0.25$ ). K-means and hierarchical clustering are executed on both the signal and the ratio data for all genes and classes. Plots of expression profiles and a correlation matrix are made for each Class group, which will show the relation of the genes within each Class group. Finally, a whole set of tab-delimited tables are produced for further downstream analyses or for drawing graphs in other programs, as required.

\section{Gene network}

Venn diagrams are traditionally used to show the overlap between experiments, but this way of presenting limits the number of experiments that can be included. T-REx circumvents this problem and shows overlap between an unlimited number of experiments in a gene network using the Reingold-Tilford layout [12]. Furthermore, this gene network is exported as a table of nodes and edges that can be examined in a gene network analysis program such as Cytoscape [13].

\section{Gene set enrichment analysis}

The results of the RNA-seq statistical pipeline describe and analyze the transcriptional behavior of genes/RNAs independently of the organism under study. To add organism-specific information to the analyzed data, a Gene Set Enrichment Analysis (GSEA) [14] is commonly used to unravel the bigger biological picture. The main issue in such an analysis is the availability of classification data for the specific organism such as Gene Ontology (GO) and metabolic pathways (KEGG). To allow for easy GSEA we (re-)annotated all proteins of all publically available bacterial complete genomes for GO, InterPro, KEGG, MetaCyc, PFAM domains, Superfamily and Gene3D. We implemented a webserver allowing to use this classification data for GSEA (http://genome2d.molgenrug.nl/index.php/functional-analysis-go-ipr).

The workflow of T-REx is presented in Fig. 1. The analysis pipeline has been written in $R$ [15] and is freely available on request. For reasons of user-friendliness, we offer a web server for uploading of the data files. The results of the analyses can be mined on a web-browser or downloaded as a zip-file (containing all html files, images and data files) for later use. Depending on server demand, a full analysis takes around $1 \mathrm{~min}$.

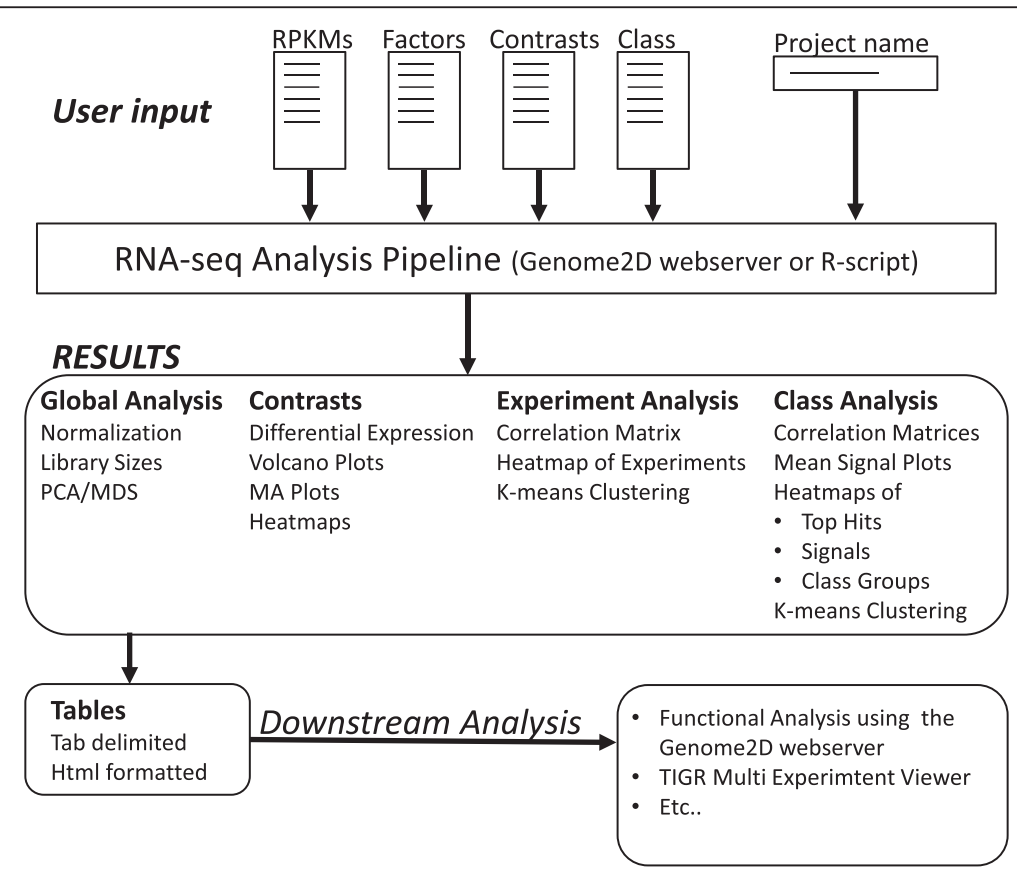

Figure 1 Flow chart of the RNA-seq analysis pipeline. User input consists of the four data files defined in Table 1 and a project name. Parameters such as thresholds, $p$-value cutoffs and k-means settings are predefined or will be estimated by the analysis pipeline 


\section{Results and discussion}

The performance of the RNA-seq data analysis pipeline was assessed using the RNA-seq expression dataset (DatasetS1) of Brinsmade and coworkers [16]. These authors performed RNA-seq to study the effect of three separate single amino acid changes in the global transcription factor for nitrogen metabolism CodY of Bacillus subtilis 168. The format of DatasetS1 could be directly used as an input for our RNA-seq analysis pipeline. A Factors file was created to define strains and replicates, as explained in Table 1a. To find differential gene expression, all three CodY mutants were compared to the wild-type, as defined by the Contrasts file (Table 1B). Apart from the Data table, a Factors file and a Contrasts file, a Class file was made containing information on the CodY regulon and two other interesting regulons: those of CcpA $[17,18]$ and ArgR [19] (Table 1C). The four files were used as inputs for the webserver and over $40 \mathrm{html}$ pages were retrieved that refer to 104 graphs, 45 tables and one html overview table (see Additional file 3).

\section{Global analysis}

The results of the global analysis (Fig. 2) showed that the library sizes and signal distributions were comparable for all samples. The biological replicates of the CodY mutant F71Y showed a higher correlation than those of the other 2 mutants, $\mathrm{R} 61 \mathrm{~K}$ and $\mathrm{R} 61 \mathrm{H}$, but all samples were well distributed upon examination of the Multi-Dimensional Scaling (MDS) plot (Fig. 2).

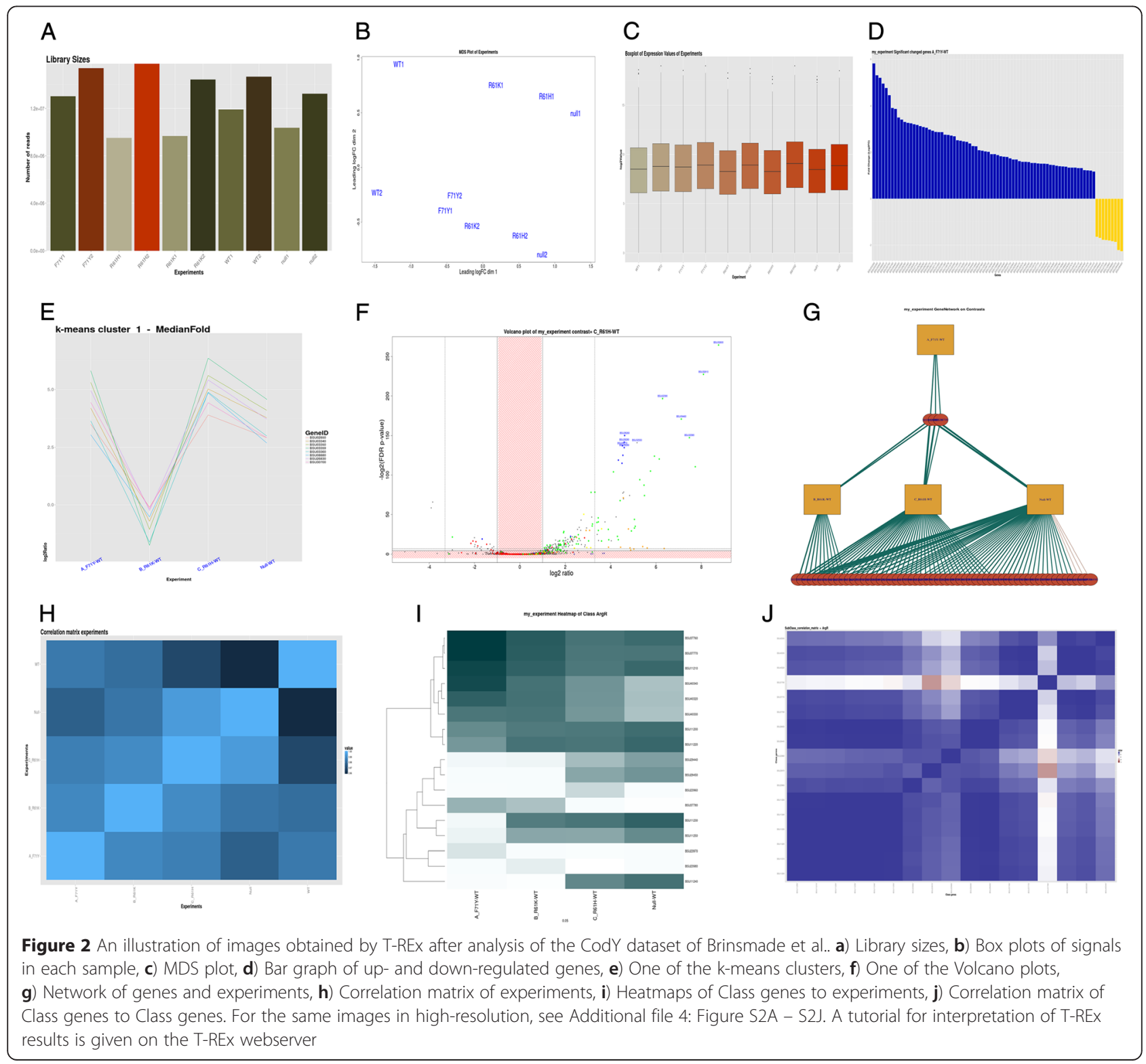




\section{Contrasts analysis}

Similar to what was observed by Brinsmade et al., more genes were up- than down-regulated in the $\operatorname{CodY}$ knock-out (Null mutant) versus wild-type comparison at a $p$-value $<0.05$ and a fold-change $>3$. The absolute number of differentially expressed genes was higher in our analysis as we used a Fold Change cutoff of 2 instead of 3 (Table 2D, $p$-value $<0.05$ and fold-change $>2$ ). Genes of the known CodY regulon were colored green (see the Class file of the example data) allowing to easily spot them in the MA- and volcano plots. Thus, it is immediately clear that more genes than only those of the CodY regulon are differentially expressed, as was also pinpointed by Brinsmade et al.. The blue dots in the upper-right quadrant of the volcano plots indicate that several genes of the CodY regulon are also part of the CcpA regulon, an observation that was also reported in Brinsmade et al.. The heatmap (Additional file 4: Figure S2I) of differentially expressed genes in all CodY mutants showed interesting groupings of genes, which might be of importance for biological interpretation and further analysis.

\section{Experiment analysis}

When analyzing all CodY mutants (targets) to the wild type (control), the number of up and down regulated genes identified by T-REx was comparable to that reported in Brinsmade et al. (Table 2). The 'Correlation Matrix of Experiments' figure (Fig. 2 and Additional file 4: Figure $\mathrm{S} 2 \mathrm{H}$ ) is in agreement with a gradual increase in the number of differentially expressed genes in the mutants, in the order F71Y, R61K, R61H and Null, as was also concluded in Brinsmade et al.. Automated k-means clustering of differentially expressed genes in the various mutants (see Additional file 5: Figure S3) also shows a gradient of gene expression, suggesting that not only the number of differentially expressed genes differs between the mutants, but also their gene expression levels. By a gradual increase in expression of certain genes in the various mutants, the ratios (mutant versus WT) pass a

Table 2 Overview table of the analysis of differential gene expression

\begin{tabular}{llll}
\hline Contrast & Total number of genes & Up-regulated & Down-regulated \\
\hline A_F71Y-WT & 4176 & 72 & 9 \\
B_R61K-WT & 4176 & 126 & 15 \\
C_R61H-WT & 4176 & 219 & 25 \\
Null-WT & 4176 & $282(196 / 212)$ & $47(27 / 29)$ \\
\hline
\end{tabular}

The numbers of up- and down-regulated genes were determined using default cutoffs $p$-value $\leq 0.05$ and fold-change $\geq 2$. Within brackets $p$-value $\leq 0.05$ and fold change $\geq 3$ as was mentioned in Brinsmade et al. and our pipeline, respectively preset threshold value, which might explain why the number of differentially expressed genes increases.

\section{Class analysis}

The T-REx pipeline performs an in-depth analysis on classes pre-defined by the user. Here we defined five classes; the regulons CodY, CcpA, ArgR, MalR and a Class of genes that are under control of both CodY and CcpA. The heatmap (Class genes to experiments) and the correlation matrix of Class genes showed that some members of the known CodY regulon do not have a good correlation over the experiments. Brinsmade et al. excluded several genes from their analyses because of their complex gene expression patterns. To study this phenomenon we added these genes in a separate Class 'Complex' and colored them orange. The volcano plots showed that a subset of 7 of these genes appears as differentially regulated compared to the wild type strain in three of the four contrasts. The probability of their differential expression is close to the $p$-value threshold (in this case 0.05) in the mutant R61K. The heatmap of signals of Class 'Complex' (see Additional file 6: Figure S4A) showed that these 7 genes have a gene expression pattern that is different from the genes of the CodY regulon (see Additional file 6: Figure S4B).

\section{Conclusion}

The parameter-free RNA-seq analysis pipeline T-REx is a fast, easy to use and comprehensive way to perform statistical analysis of gene expression data derived from RNA-seq data. Typical graphics and tables are automatically generated, which enables a direct overview of the biological relevance of the data, obviating laborious combining and complex filtering operations of data. Furthermore, T-REx produces data tables for (optional) downstream processing. The case study presented in this article compared the analyses performed by Brinsmade et al. and T-REx. The outcome shows that T-REx can quickly and fully automatically perform statistical analyses on gene expression data derived from RNA-seq. It reproduced the results of the original study without requiring additional statistical analyses. The T-REx pipeline is continuously updated and expanded to fully utilize the potential of RNA-seq gene expression data sets.

\section{Availability and requirements}

- Project name: T-REx.

- Project home page: http://genome2d.molgenrug.nl.

- Operating system(s): Platform independent.

- Programming language: Perl, R.

- License: This website is free and open to all users and there is no login requirement. 


\section{Additional files}

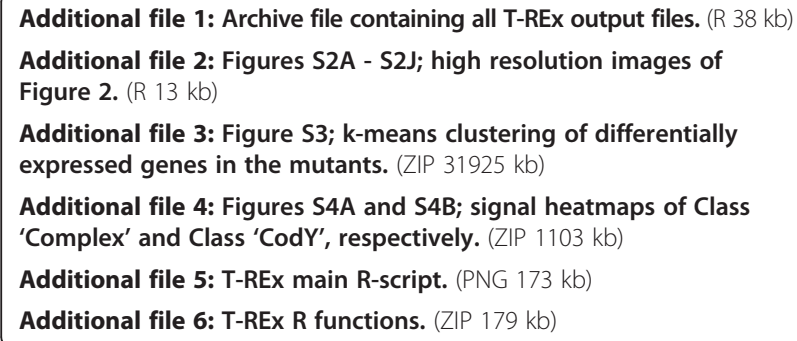

\section{Competing interests}

The authors declare that they have no competing interests.

\section{Authors' contributions}

AJ developed and designed the webserver. SM participated in the improvement of the webserver functionalities. AJ and SM drafted the manuscript and OPK and JK revised it. All authors have read and approved the final submitted version of this manuscript.

\section{Acknowledgements}

We are grateful to Martijn Herber for his support on server management and Jimmy Omony for suggestions on the R script.

\section{Author details}

${ }^{1}$ Molecular Genetics, University of Groningen, Nijenborgh 7, 9747AG Groningen, The Netherlands. ${ }^{2}$ Top Institute Food and Nutrition (TIFN), Nieuwe Kanaal 9A, 6709PA, Wageningen, The Netherlands. ${ }^{3}$ Molecular Genetics, Groningen Biomolecular Sciences and Biotechnology Institute, University of Groningen, Groningen, The Netherlands.

Received: 14 April 2015 Accepted: 7 August 2015

Published online: 03 September 2015

\section{References}

1. Ramirez-Gonzalez R, Bonnal RJ, Caccamo M, MacLean D. Bio-samtools: ruby bindings for SAMtools, a library for accessing BAM files containing high-throughput sequence alignments. Source Code Biol Med. 2012;7:6.

2. Quinlan AR. BEDTools: the swiss-army tool for genome feature analysis. Curr Protoc Bioinformatics. 2014;47:11.12. 1-11.12. 34.

3. McClure R, Balasubramanian D, Sun Y, Bobrovskyy M, Sumby P, Genco CA et al. Computational analysis of bacterial RNA-Seq data. Nucleic Acids Res. 2013;41(14):e140.

4. Boria I, Boatti L, Pesole G, Mignone F. NGS-Trex: Next Generation Sequencing Transcriptome profile explorer. BMC Bioinformatics. 2013;14 Suppl 7:S10-2105-14-S7-S10. Epub 2013 Apr 22.

5. Robinson MD, McCarthy DJ, Smyth GK. edgeR: a Bioconductor package for differential expression analysis of digital gene expression data. Bioinformatics. 2010;26(1):139-40.

6. Anders $\mathrm{S}$, Huber W. Differential expression analysis for sequence count data. Genome Biol. 2010;11(10):R106.

7. Soneson C, Delorenzi M. A comparison of methods for differential expression analysis of RNA-seq data. BMC Bioinformatics. 2013;14:91-2105-14-91.

8. Smyth GK: Limma: linear models for microarray data. In Bioinformatics and computational biology solutions using $\mathrm{R}$ and Bioconductor. Edited by Anonymous Springer: Statistics for Biology and Health. 2005:397-420.

9. Huber W, Carey VJ, Gentleman R, Anders S, Carlson M, Carvalho BS, et al. Orchestrating high-throughput genomic analysis with Bioconductor. Nat Methods. 2015;12(2):115-21.

10. Li B, Dewey CN. RSEM: accurate transcript quantification from RNA-Seq data with or without a reference genome. BMC Bioinformatics. 2011;12:323-2105-12-323

11. MacQueen JB. Some Methods for classification and Analysis of Multivariate Observations. Proceedings of 5th Berkeley Symposium on Mathematical Statistics and Probability 1 University of California Press 1967, (1):281-297.
12. Reingold EM, Tilford JS. Tidier drawings of trees. IEEE Transactions on Software Engineering archive. 1981;7(2):223-8.

13. Kohl M, Wiese S, Warscheid B. Cytoscape: software for visualization and analysis of biological networks. Methods Mol Biol. 2011;696:291-303.

14. Subramanian A, Tamayo P, Mootha VK, Mukherjee S, Ebert BL, Gillette MA, et al. Gene set enrichment analysis: a knowledge-based approach for interpreting genome-wide expression profiles. Proc Natl Acad Sci U S A. 2005;102(43):15545-50.

15. R: A language and environment for statistical computing. [http://www.R-project.org/].

16. Brinsmade SR, Alexander EL, Livny J, Stettner Al, Segre D, Rhee KY, et al. Hierarchical expression of genes controlled by the Bacillus subtilis global regulatory protein CodY. Proc Natl Acad Sci U S A. 2014;111(22):8227-32

17. Henkin TM. The role of CcpA transcriptional regulator in carbon metabolism in Bacillus subtilis. FEMS Microbiol Lett. 1996;135(1):9-15.

18. Moreno MS, Schneider BL, Maile RR, Weyler W, Saier Jr MH. Catabolite repression mediated by the CcpA protein in Bacillus subtilis: novel modes of regulation revealed by whole-genome analyses. Mol Microbiol. 2001;39(5):1366-81.

19. Sonenshein AL. Control of key metabolic intersections in Bacillus subtilis. Nat Rev Microbiol. 2007;5(12):917-27.

\section{Submit your next manuscript to BioMed Central and take full advantage of:}

- Convenient online submission

- Thorough peer review

- No space constraints or color figure charges

- Immediate publication on acceptance

- Inclusion in PubMed, CAS, Scopus and Google Scholar

- Research which is freely available for redistribution

Submit your manuscript at www.biomedcentral.com/submit 